\title{
Bio-efficacy of Certain Selective Insecticides against Whitefly, (Bemisia tabaci) on Mungbean, (Vigna radiata L., Wilczek)
}

\section{Nadeya Khaliq, Virender Koul, Uma Shankar, Suheel Ahmad Ganai* and Thanlass Norboo}

\author{
Block No. 6, Division of Entomology, FoA, Main Campus, SKUAST-J, Chatha, \\ Jammu -180 009, J\&K, India \\ *Corresponding author
}

\section{A B S T R A C T}

\begin{tabular}{|l|}
\hline Key w or d s \\
Bemisia tabaci, \\
Vigna radiata, \\
Management, \\
Imidacloprid, \\
NSKE. \\
\hline Article Info \\
\hline $\begin{array}{l}\text { Accepted: } \\
\text { 26 June 2017 } \\
\text { Available Online: } \\
\text { 10 July 2017 }\end{array}$ \\
\hline
\end{tabular}

A field experiment was conducted on mungbean to evaluate certain selective insecticidal molecules, viz., imidacloprid, dimethoate + triazophos, thiamethoxam, neem oil, dimethoate, oxy-demeton methyl, triazophos and NSKE against the field populations of $B$. tabaci, during Zaid and Kharif season of the year 2014. The results indicated that imidacloprid showed higher efficacy against $B$. tabaci in reducing pest population followed by dimethoate + triazophos. Triazophos and NSKE were found to be least effective.

\section{Introduction}

India has distinction of being world's largest producer of grain legumes by growing over a dozen of pulse crops. Legumes occupy an important place in human nutrition, especially among the low-income groups of people in developing countries (Osorio-Diaz et al., 2003). India grows the largest varieties of pulses in the world accounting for about $32 \%$ of the area and $23 \%$ of the world production and thus holds the first rank in pulses production and consumption. Pulses production has registered a remarkable increase from 14.76 million tonnes in 2007-08 to a record level of 18.24 million tonnes in 2010-11. Productivity of pulses has increased from $625 \mathrm{~kg}$ per hectare in $2007-08$ to $699 \mathrm{~kg}$ per hectare in 2011-12. Amongst Kharif pulses, Mungbean, Vigna radiata (L.) Wilczek is one of the major pulse crops. India is the biggest producer of mung bean having about 3 million hectare cultivated land with a production of 1.42 million tonnes (Singh and Ahlawat, 2005). It is widely cultivated in various states of India and extensively grown in Uttar Pradesh, Madhya Pradesh, Rajasthan, Maharashtra, Orissa, Karnataka, Andhra Pradesh, Gujarat, Bihar, Haryana and Delhi during Kharif and Zaid season.

The losses due to insects and non-insect pests to pulses are of the major single factor responsible low yields as low as 5-6 q/ha (Lal 
et al., 1980). In Jammu subtropics, 38 insect pests have been recorded, out of which 22 are regular visitors (Tikoo, 1996). The economically important pests are whitefly, Bemisia tabaci Gennadius; aphid, Aphis craccivora Koch; pod borer, Maruca testulalis Geyer; and Bihar hairy caterpillar, Spilosoma oblique Walker. Among these $B$. tabaci (Insecta: Hemiptera: Aleyrodidae) is an important cosmopolitan and polyphagous species. Bemisia tabici is a phloem feeding insect and causing damage directly through feeding and indirectly through the transmission of plant pathogenic viruses, primarily begomoviruses (Oliveira et al., 2001). Yellow mosaic virus causes irregular yellow and green patches on older leaves. Affected plants produce less number of pods and flowers and few seeds. It can cause yield losses of 30 to $70 \%$ (Marimuthu et al., 1981).

Since whitefly infestations can easily go unnoticed until they reach high numbers, it is important to minimize potential infestations by employing all possible control tactics prior to chemical control. Initially all whitefly stages typically occupy the underside of leaves of infested plants. The more susceptible stages are the younger nymphs. The later stages, such as the fourth instar and red eye stage, are more difficult to control. Therefore, the best control is achieved when a maximum effort is made to contact the underside of leaves with repeated spray applications targeting the earlier, susceptible stages for a period of about three weeks. Heavier infestations may require more applications for a more extended period of time. Contact insecticides, including soaps and oils, may be more effective early in the growth cycle when the foliage is less dense and contact with young nymphs is more likely. Later in the cropping cycle, when dense foliage is present, a systemically acting material may be more efficient. In order to maintain the pest population under the economical harming level, the treatments made beginning with the phase of seedlings have the role to avoid rapid multiplication of the fly. However, whitefly chemical control is challenging because it causes natural enemies mortality (Michaud and McKenzie, 2004) and insecticide resistance development to divergent classes of insecticides, especially organophosphates, pyrethroids, cyclodienes and relatively new group of chloro-nicotinyl insecticides. Insecticide resistance in whiteflies is well documented as a widespread phenomenon in those countries in which monitoring of resistance has been conducted (Denholm et al., 2003). Hence there is need to use newer insecticides, which are selective and are also effective at low dose. The neonicotinoid insecticide, imidacloprid and the growth-regulating insecticides (IGRs), buprofezin and pyriproxyfen serve critical role in controlling whiteflies (B. tabaci) in arid regions of the world (Denholm et al., 1998). In Jammu \& Kashmir, no such work has been done on these aspects. Therefore, keeping in view the economic importance of the crop and the magnitude of the damage caused by insect pests, the present study has been proposed.

\section{Materials and Methods}

Experiments were conducted at the Research field, Division of Entomology, Main Campus, Chatha, Jammu during Zaid and Kharif season of the year 2014. The seeds were sown under natural conditions without the application of any manures and fertilizer. The experiments were laid out in randomized block design (RBD) with three replications of each treatment. In view of severity of damage caused by whitefly on mungbean and mark available effective control measures, field trails on mungbean varieties were conducted. In all there were 9 treatments each replicated three times including an untreated control and their details are given below. 
The mungbean varieties were sown with $30 \mathrm{~cm} \times 10 \mathrm{~cm}$ spacing in the plot size of $3 \times 2 \mathrm{~m}$. The treatments were given to the crop with help of knapsack sprayer. For recording observations, three leaves one each from top, middle and bottom were earmarked in each plot and observations were recorded at $24 \mathrm{hrs}$ before spray and then 1 day, 3 days, 7 days and 14 days after spray. Spray was applied when whitefly population reaches 1-5 per leaf.

\section{Results and Discussion}

\section{Zaid, 2014}

Efficacy of the insecticides for the management of the pest revealed that all the insecticidal treatments at 1, 3, 7 and 14 days after spray were superior to control in reducing the pest population (Table 1). The data recorded on $1^{\text {st }}$ day after spray revealed that imidacloprid (1.71 whitefly/plant) was found to be most effective treatment in reducing the whitefly population which was at par with dimethoate + triazophos (1.98 whitefly/plant). The next best treatment was thiamethoxam (3.00 whitefly/plant), neem oil (3.33 whitefly/plant), dimethoate (3.68 whitefly/plant) oxy-demeton methyl (4.00 whitefly/plant), triazophos (4.36 whitefly/plant), which were at par with each other. NSKE (4.67 whitefly/plant) was found to be least effective against the whitefly. The observations recorded on $3^{\text {rd }}$ day after spray revealed imidacloprid (1.33 whitefly/plant) was found to be most effective treatment in reducing the whitefly population which was at par with dimethoate + triazophos (1.65 whitefly/plant). The next best treatment was thiamethoxam (2.68 whitefly/plant), neem oil (3.00 whitefly/plant), dimethoate (3.33 whitefly/plant), oxy-demeton methyl (3.70 whitefly/plant), which were at par with each other. Triazophos (4.00 whitefly/plant) and NSKE (4.23 whitefly/plant) were found to be least effective against the whitefly. On $7^{\text {th }}$ day after spray imidacloprid (2.00 whitefly/plant) was found to be most effective treatment in reducing the whitefly population which was at par with dimethoate + triazophos (2.71 whitefly/plant). The next best treatment was thiamethoxam (3.36 whitefly/plant), neem oil (3.67 whitefly/plant), dimethoate (4.01whitefly/plant), oxy-demeton methyl (4.35 whitefly/plant), which were at par with each other. Triazophos (4.72 whitefly/plant) was found to be least effective against the whitefly followed by NSKE (5.00 whitefly/plant). The observations on $14^{\text {th }}$ day after spray revealed that imidacloprid (2.69 whitefly/plant) was found to be most effective treatment in reducing the whitefly population which was at par with dimethoate + triazophos (3.35 whitefly/plant). The next best treatment was thiamethoxam $\quad(4.00$ whitefly/plant), neem oil $\quad(4.38$ whitefly/plant), dimethoate (4.70 whitefly/plant), oxy-demeton methyl (5.42 whitefly/plant), which were at par with each other. Triazophos (6.00 whitefly/plant) and NSKE (6.83 whitefly/plant) were found to be least effective against the whitefly.

\section{Kharif, 2014}

\section{First spray}

The data regarding management of whitefly, Bemisia tabaci during Kharif 2014 (Table 2) revealed that the observations recorded on $1^{\text {st }}$ day after spray revealed that all the treatments proved significantly superior over control. Imidacloprid (8.00 whitefly/plant) was found to be most effective treatment in reducing the whitefly population which was at par with dimethoate + triazophos (8.69 whitefly/plant). The next best treatment was thiamethoxam (10.00 whitefly/plant), neem oil (11.36 whitefly/plant), dimethoate (13.00 whitefly/plant), triazophos (14.33 whitefly/plant) and oxy-demeton methyl 
(16.00 whitefly/plant), which were at par with each other. NSKE (18.67 whitefly/plant) was found to be least effective against the whitefly. The observations recorded on $3^{\text {rd }}$ day after spray revealed that all the treatments proved significantly superior over control. Imidacloprid (6.69 whitefly/plant) was found to be most effective treatment in reducing the whitefly population which was at par with dimethoate + triazophos (7.34 whitefly/plant). The next best treatment was thiamethoxam (8.67 whitefly/plant), neem oil (10.00 whitefly/plant), dimethoate (12.00 whitefly/plant), triazophos (12.67 whitefly/plant), which were at par with each other. Oxy-demeton methyl (14.00 whitefly/plant) and NSKE (16.68 whitefly/plant) were found to be least effective against the whitefly. The observations recorded on $7^{\text {th }}$ day after spray revealed that all the treatments proved significantly superior over control. Imidacloprid (6.00 whitefly/plant) was found to be most effective treatment in reducing the whitefly population which was at par with dimethoate + triazophos (6.67 whitefly/plant). The next best treatment was thiamethoxam (8.00 whitefly/plant), neem oil $(9.00$ whitefly/plant), dimethoate (11.33 whitefly/plant), triazophos (11.68 whitefly/plant), which were at par with each other. Oxy-demeton methyl (12.68 whitefly/plant) was found to be least effective against the whitefly followed by NSKE (15.70 whitefly/plant). On $14^{\text {th }}$ day after spray revealed that all the treatments proved significantly superior over control. Imidacloprid (6.98 whitefly/plant) was found to be most effective treatment in reducing the whitefly population which was at par with dimethoate + triazophos (7.69 whitefly/plant). The next best treatment was thiamethoxam (9.00 whitefly/plant), neem oil (10.34 whitefly/plant), dimethoate (12.05 whitefly/plant), triazophos (12.78 whitefly/plant), which were at par with each other. Oxy-demeton methyl (13.33 whitefly/plant) and NSKE (17.00 whitefly/plant) were found to be least effective against the whitefly.

Treatments each replicated three times including an untreated control and their details

\begin{tabular}{|c|c|c|}
\hline S.No. & Treatments & Dose/Concentration \\
\hline $\mathbf{T}_{\mathbf{1}}$ & Dimethoate 30 EC & $0.03 \%$ \\
\hline $\mathbf{T}_{\mathbf{2}}$ & Dimethoate 30 EC+Triazophos 40 EC & $\begin{array}{c}\text { Seed treatment @ 5ml/kg }+0.04 \% \\
\text { foliar spray respectively }\end{array}$ \\
\hline $\mathbf{T}_{\mathbf{3}}$ & Oxydemeton methyl 25 EC & $0.03 \%$ \\
\hline $\mathbf{T}_{\mathbf{4}}$ & Imidacloprid 17.8 SL & $\begin{array}{c}\text { Seed treatment @ 3 ml / kg + 2 } \\
\text { sprays @ 0.008 \% at 15 days } \\
\text { interval }\end{array}$ \\
\hline $\mathbf{T}_{\mathbf{5}}$ & Triazophos 40 EC & \begin{tabular}{c}
$0.04 \%$ \\
\hline $\mathbf{T}_{\mathbf{6}}$
\end{tabular} \\
\hline $\mathbf{T}_{\mathbf{7}}$ & NSKE & $5 \%$ \\
\hline $\mathbf{T}_{\mathbf{8}}$ & Thiamethoxam 25WG & $0.0125 \%$ \\
\hline $\mathbf{T}_{\mathbf{9}}$ & T8 Neem oil & $0.15 \%$ \\
\hline
\end{tabular}


Table.1 Bio-efficacy of certain selective insecticides against whitefly on mungbean during, Zaid season 2014

\begin{tabular}{|c|c|c|c|c|c|c|c|}
\hline \multirow[t]{2}{*}{ S.No. } & \multirow[t]{2}{*}{ Treatments } & \multirow{2}{*}{$\begin{array}{l}\text { Concentration } \\
\text { /Dose }\end{array}$} & \multicolumn{5}{|c|}{ Mean whitefly population/plant } \\
\hline & & & 1DBS* & $1 \mathrm{DAS} *$ & $3 \mathrm{DAS}$ & 7DAS & 14DAS \\
\hline $\mathbf{T}_{1}$ & Dimethoate $30 \mathrm{EC}$ & $0.03 \%$ & $\begin{array}{c}6.68 \\
(2.76)\end{array}$ & $\begin{array}{c}3.68 \\
(2.13)\end{array}$ & $\begin{array}{c}3.33 \\
(2.08)\end{array}$ & $\begin{array}{c}4.01 \\
(2.24)\end{array}$ & $\begin{array}{c}4.70 \\
(2.37)\end{array}$ \\
\hline $\mathbf{T}_{2}$ & $\begin{array}{c}\text { Dimethoate } 30 \\
\text { EC+Triazophos } 40 \text { EC }\end{array}$ & $\begin{array}{l}\text { Seed treatment @ } \\
5 \mathrm{ml} / \mathrm{kg}+0.04 \% \\
\text { foliar spray } \\
\text { respectively }\end{array}$ & $\begin{array}{l}4.67 \\
(2.38)\end{array}$ & $\begin{array}{c}1.98 \\
(1.70)\end{array}$ & $\begin{array}{c}1.65 \\
(1.69)\end{array}$ & $\begin{array}{c}2.71 \\
(1.91)\end{array}$ & $\begin{array}{l}3.35 \\
(2.08)\end{array}$ \\
\hline $\mathbf{T}_{\mathbf{3}}$ & Oxydemeton methyl $25 \mathrm{EC}$ & $0.03 \%$ & $\begin{array}{c}6.00 \\
(2.34)\end{array}$ & $\begin{array}{c}4.00 \\
(2.26)\end{array}$ & $\begin{array}{c}3.70 \\
(2.16)\end{array}$ & $\begin{array}{c}4.35 \\
(2.31)\end{array}$ & $\begin{array}{c}5.42 \\
(2.54)\end{array}$ \\
\hline $\mathbf{T}_{4}$ & Imidacloprid 17.8 SL & $\begin{array}{c}\text { Seed treatment @ } \\
3 \mathrm{ml} / \mathrm{kg}+2 \\
\text { sprays @ } 0.008 \% \\
\text { at } 15 \text { days interval }\end{array}$ & $\begin{array}{l}4.33 \\
(2.30)\end{array}$ & $\begin{array}{c}1.71 \\
(1.68)\end{array}$ & $\begin{array}{l}1.33 \\
(1.52)\end{array}$ & $\begin{array}{c}2.00 \\
(1.73)\end{array}$ & $\begin{array}{l}2.69 \\
(1.90)\end{array}$ \\
\hline $\mathbf{T}_{\mathbf{5}}$ & Triazophos $40 \mathrm{EC}$ & $0.04 \%$ & $\begin{array}{c}6.67 \\
(2.76)\end{array}$ & $\begin{array}{c}4.36 \\
(2.29)\end{array}$ & $\begin{array}{c}4.00 \\
(2.23)\end{array}$ & $\begin{array}{c}4.72 \\
(2.38)\end{array}$ & $\begin{array}{c}6.00 \\
(2.65)\end{array}$ \\
\hline $\mathbf{T}_{6}$ & NSKE $5 \%$ & $5 \%$ & $\begin{array}{c}6.04 \\
(2.64)\end{array}$ & $\begin{array}{c}4.67 \\
(2.38)\end{array}$ & $\begin{array}{c}4.23 \\
(2.29)\end{array}$ & $\begin{array}{c}5.00 \\
(2.45)\end{array}$ & $\begin{array}{c}6.83 \\
(2.79)\end{array}$ \\
\hline $\mathbf{T}_{7}$ & Thiamethoxam 25WG & $0.0125 \%$ & $\begin{array}{c}6.34 \\
(2.69) \\
\end{array}$ & $\begin{array}{c}3.00 \\
(2.00) \\
\end{array}$ & $\begin{array}{c}2.68 \\
(1.88) \\
\end{array}$ & $\begin{array}{c}3.36 \\
(2.09) \\
\end{array}$ & $\begin{array}{c}4.00 \\
(2.24) \\
\end{array}$ \\
\hline $\mathbf{T}_{8}$ & Neem oil & $0.15 \%$ & $\begin{array}{c}6.33 \\
(2.71)\end{array}$ & $\begin{array}{c}3.33 \\
(2.06)\end{array}$ & $\begin{array}{c}3.00 \\
(2.00)\end{array}$ & $\begin{array}{c}3.67 \\
(2.13)\end{array}$ & $\begin{array}{c}4.38 \\
(2.30)\end{array}$ \\
\hline $\mathbf{T}_{9}$ & Water (Control) & - & $\begin{array}{c}6.67 \\
(2.76)\end{array}$ & $\begin{array}{c}6.00 \\
(2.65)\end{array}$ & $\begin{array}{c}6.67 \\
(2.76)\end{array}$ & $\begin{array}{c}6.70 \\
(2.77)\end{array}$ & $\begin{array}{c}7.89 \\
(2.94)\end{array}$ \\
\hline & C.D at $5 \%$ & - & N.S. & 0.484 & 0.394 & 0.403 & 0.375 \\
\hline & SE(m) & & 0.155 & 0.160 & 0.130 & 0.133 & 0.124 \\
\hline
\end{tabular}

*DBS - Days before spray and DAS - Days after spray Values in parenthesis are square root transformed 
Table.2 Bio-efficacy of certain selective insecticides against whitefly on mungbean during, Kharif season 2014

\begin{tabular}{|c|c|c|c|c|c|c|c|c|c|c|c|c|}
\hline \multirow{3}{*}{$\begin{array}{l}\text { S.N } \\
\text { o. }\end{array}$} & \multirow[t]{3}{*}{ Treatments } & \multirow{3}{*}{$\begin{array}{c}\text { Concentration/ } \\
\text { Dose }\end{array}$} & \multicolumn{10}{|c|}{ Mean whitefly population/plant } \\
\hline & & & \multicolumn{5}{|c|}{$1^{\text {st }}$ spray } & \multicolumn{5}{|c|}{$2^{\text {nd }}$ spray } \\
\hline & & & $1 \mathrm{DBS} *$ & $\begin{array}{c}\text { 1DAS } \\
*\end{array}$ & 3DAS & 7DAS & $\begin{array}{c}\text { 14DA } \\
\mathrm{S}\end{array}$ & 1DBS & 1DAS & 3DAS & 7DAS & 14DAS \\
\hline $\mathbf{T}_{1}$ & Dimethoate $30 \mathrm{EC}$ & $0.03 \%$ & $\begin{array}{l}22.33 \\
(4.83)\end{array}$ & $\begin{array}{l}13.00 \\
(3.74)\end{array}$ & $\begin{array}{l}12.00 \\
(3.60)\end{array}$ & $\begin{array}{l}11.33 \\
(3.51)\end{array}$ & $\begin{array}{l}12.05 \\
(3.60)\end{array}$ & $\begin{array}{l}13.36 \\
(3.77)\end{array}$ & $\begin{array}{l}12.00 \\
(3.61)\end{array}$ & $\begin{array}{l}10.67 \\
(3.41)\end{array}$ & $\begin{array}{l}10.00 \\
(3.31)\end{array}$ & $\begin{array}{c}9.00 \\
(3.14)\end{array}$ \\
\hline $\mathbf{T}_{2}$ & $\begin{array}{c}\text { Dimethoate } 30 \\
\text { EC+Triazophos } 40 \\
\text { EC }\end{array}$ & $\begin{array}{l}\text { Seed treatment @ } \\
5 \mathrm{ml} / \mathrm{kg}+0.04 \% \\
\text { foliar spray } \\
\text { respectively }\end{array}$ & $\begin{array}{l}21.00 \\
(4.69)\end{array}$ & $\begin{array}{l}8.69 \\
(3.10)\end{array}$ & $\begin{array}{l}7.34 \\
(2.88)\end{array}$ & $\begin{array}{c}6.67 \\
(2.76)\end{array}$ & $\begin{array}{l}7.69 \\
(2.94)\end{array}$ & $\begin{array}{c}9.00 \\
(3.17)\end{array}$ & $\begin{array}{c}6.68 \\
(2.75)\end{array}$ & $\begin{array}{c}5.66 \\
(2.56)\end{array}$ & $\begin{array}{l}4.33 \\
(2.30)\end{array}$ & $\begin{array}{l}3.32 \\
(2.06)\end{array}$ \\
\hline $\mathbf{T}_{3}$ & $\begin{array}{l}\text { Oxydemeton methyl } \\
25 \mathrm{EC}\end{array}$ & $0.03 \%$ & $\begin{array}{l}22.35 \\
(4.83)\end{array}$ & $\begin{array}{l}16.00 \\
(4.12)\end{array}$ & $\begin{array}{l}14.00 \\
(3.87)\end{array}$ & $\begin{array}{l}12.68 \\
(3.69)\end{array}$ & $\begin{array}{l}13.33 \\
(3.78)\end{array}$ & $\begin{array}{l}15.67 \\
(4.07)\end{array}$ & $\begin{array}{l}14.36 \\
(3.91)\end{array}$ & $\begin{array}{l}13.01 \\
(3.73)\end{array}$ & $\begin{array}{l}12.00 \\
(3.60)\end{array}$ & $\begin{array}{l}11.35 \\
(3.50)\end{array}$ \\
\hline $\mathbf{T}_{4}$ & $\begin{array}{c}\text { Imidacloprid } 17.8 \\
\text { SL }\end{array}$ & $\begin{array}{c}\text { Seed treatment @ } 3 \\
\mathrm{ml} / \mathrm{kg}+2 \text { sprays @ } \\
0.008 \% \text { at } 15 \text { days } \\
\text { interval }\end{array}$ & $\begin{array}{l}20.00 \\
(4.58)\end{array}$ & $\begin{array}{l}8.00 \\
(3.00)\end{array}$ & $\begin{array}{l}6.69 \\
(2.77)\end{array}$ & $\begin{array}{l}6.00 \\
(2.64)\end{array}$ & $\begin{array}{c}6.98 \\
(2.79)\end{array}$ & $\begin{array}{l}8.00 \\
(2.99)\end{array}$ & $\begin{array}{l}5.33 \\
(2.51)\end{array}$ & $\begin{array}{l}4.00 \\
(2.22)\end{array}$ & $\begin{array}{l}3.32 \\
(2.07)\end{array}$ & $\begin{array}{l}2.36 \\
(1.82)\end{array}$ \\
\hline $\mathbf{T}_{5}$ & Triazophos $40 \mathrm{EC}$ & $0.04 \%$ & $\begin{array}{l}23.67 \\
(4.95)\end{array}$ & $\begin{array}{l}14.33 \\
(3.91)\end{array}$ & $\begin{array}{l}12.67 \\
(3.69)\end{array}$ & $\begin{array}{l}11.68 \\
(3.55)\end{array}$ & $\begin{array}{l}12.78 \\
(3.70)\end{array}$ & $\begin{array}{l}14.69 \\
(3.93)\end{array}$ & $\begin{array}{l}13.00 \\
(3.74)\end{array}$ & $\begin{array}{l}11.68 \\
(3.55)\end{array}$ & $\begin{array}{l}10.68 \\
(3.41)\end{array}$ & $\begin{array}{l}10.00 \\
(3.31)\end{array}$ \\
\hline $\mathbf{T}_{6}$ & NSKE $5 \%$ & $5 \%$ & $\begin{array}{l}23.68 \\
(4.97)\end{array}$ & $\begin{array}{l}18.67 \\
(4.43)\end{array}$ & $\begin{array}{l}16.68 \\
(4.20)\end{array}$ & $\begin{array}{l}15.70 \\
(4.08)\end{array}$ & $\begin{array}{l}17.00 \\
(4.25)\end{array}$ & $\begin{array}{l}18.66 \\
(4.43)\end{array}$ & $\begin{array}{l}16.35 \\
(4.16)\end{array}$ & $\begin{array}{l}14.66 \\
(3.95)\end{array}$ & $\begin{array}{r}13.32 \\
(3.78)\end{array}$ & $\begin{array}{l}13.00 \\
(3.73)\end{array}$ \\
\hline $\mathbf{T}_{7}$ & $\begin{array}{c}\text { Thiamethoxam } \\
25 \mathrm{WG}\end{array}$ & $0.0125 \%$ & $\begin{array}{l}21.33 \\
(4.72) \\
\end{array}$ & $\begin{array}{l}10.00 \\
(3.31) \\
\end{array}$ & $\begin{array}{r}8.67 \\
(3.11) \\
\end{array}$ & $\begin{array}{r}8.00 \\
(3.00) \\
\end{array}$ & $\begin{array}{r}9.00 \\
(3.16) \\
\end{array}$ & $\begin{array}{l}10.68 \\
(3.41) \\
\end{array}$ & $\begin{array}{r}8.32 \\
(3.05) \\
\end{array}$ & $\begin{array}{r}7.00 \\
(2.82) \\
\end{array}$ & $\begin{array}{r}6.33 \\
(2.70) \\
\end{array}$ & $\begin{array}{l}6.00 \\
(2.63) \\
\end{array}$ \\
\hline $\mathbf{T}_{8}$ & Neem oil & $0.15 \%$ & $\begin{array}{l}25.00 \\
(5.10)\end{array}$ & $\begin{array}{l}11.36 \\
(3.51)\end{array}$ & $\begin{array}{l}10.00 \\
(3.31)\end{array}$ & $\begin{array}{c}9.00 \\
(3.16)\end{array}$ & $\begin{array}{l}10.34 \\
(3.36)\end{array}$ & $\begin{array}{l}12.35 \\
(3.64)\end{array}$ & $\begin{array}{l}11.00 \\
(3.46)\end{array}$ & $\begin{array}{c}9.33 \\
(3.20)\end{array}$ & $\begin{array}{c}8.36 \\
(3.05)\end{array}$ & $\begin{array}{c}7.36 \\
(2.87)\end{array}$ \\
\hline $\mathbf{T}_{9}$ & Water (Control) & - & $\begin{array}{l}23.67 \\
(4.97)\end{array}$ & $\begin{array}{l}24.68 \\
(5.07)\end{array}$ & $\begin{array}{l}25.67 \\
(5.16)\end{array}$ & $\begin{array}{l}26.69 \\
(5.26)\end{array}$ & $\begin{array}{l}28.00 \\
(5.38)\end{array}$ & $\begin{array}{l}28.34 \\
(5.41)\end{array}$ & $\begin{array}{l}27.67 \\
(5.35)\end{array}$ & $\begin{array}{l}28.00 \\
(5.38)\end{array}$ & $\begin{array}{r}28.33 \\
(5.41)\end{array}$ & $\begin{array}{l}29.33 \\
(5.50)\end{array}$ \\
\hline & C.D at $5 \%$ & - & NS & 0.295 & 0.301 & 0.316 & 0.309 & 0.477 & 0.299 & 0.391 & 0.304 & 0.547 \\
\hline & SE(m) & & 0.127 & 0.098 & 0.108 & 0.105 & 0.102 & 0.158 & 0.099 & 0.129 & 0.100 & 0.181 \\
\hline
\end{tabular}

*DBS - Days before spray and DAS - Days after spray Values in parenthesis are square root transformed 


\section{Second spray}

The data regarding management of whitefly, Bemisia tabaci during Kharif 2014 (Table 2) revealed that the observations recorded on $1^{\text {st }}$ day after spray revealed that all the treatments proved significantly superior over control. Imidacloprid (5.33 whitefly/plant) was found to be most effective treatment in reducing the whitefly population which was at par with dimethoate + triazophos (6.68 whitefly/plant). The next best treatment was thiamethoxam (8.33 whitefly/plant), neem oil (11.00 whitefly/plant), dimethoate $\quad(12.00$ whitefly/plant), triazophos (13.00 whitefly/plant) and oxy-demeton methyl (14.36 whitefly/plant), which were at par with each other. NSKE (16.35 whitefly/plant) was found to be least effective against the whitefly. The observations recorded on $3^{\text {rd }}$ day after spray revealed that all the treatments proved significantly superior over control. Imidacloprid (4.00 whitefly/plant) was found to be most effective treatment in reducing the whitefly population which was at par with dimethoate + triazophos (5.66 whitefly/plant). The next best treatment was thiamethoxam (7.00 whitefly/plant), neem oil (9.33 whitefly/plant), dimethoate (10.67 whitefly/plant), triazophos (11.68 whitefly/plant), which were at par with each other. Oxy-demeton methyl (13.01 whitefly/plant) and NSKE (14.66 whitefly/plant) were found to be least effective against the whitefly. The observations recorded on $7^{\text {th }}$ day after spray revealed that all the treatments proved significantly superior over control. Imidacloprid (3.32 whitefly/plant) was found to be most effective treatment in reducing the whitefly population which was at par with dimethoate + triazophos (4.33 whitefly/plant). The next best treatment was thiamethoxam (6.33 whitefly/plant), neem oil (8.36 whitefly/plant), dimethoate (10.00 whitefly/plant), triazophos $\quad(10.68$ whitefly/plant), which were at par with each other. Oxy-demeton methyl (12.00 whitefly/plant) was found to be least effective against the whitefly followed by NSKE (13.32 whitefly/plant). On $14^{\text {th }}$ day after spray revealed that all the treatments proved significantly superior over control. Imidacloprid (2.36 whitefly/plant) was found to be most effective treatment in reducing the whitefly population which was at par with dimethoate + triazophos (3.32 whitefly/plant). The next best treatment was thiamethoxam (6.00 whitefly/plant), neem oil (7.36 whitefly/plant), dimethoate (9.00 whitefly/plant), triazophos (10.00 whitefly/plant), which were at par with each other. Oxy-demeton methyl (11.35 whitefly/plant) and NSKE (13.00 whitefly/plant) were found to be least effective against the whitefly. The efficacy and superiority of imidacloprid was observed in the present study is in line with the results obtained by Mohan and Katiyar (2000) who reported that imidacloprid was the most effective in suppressing the whitefly population and Dandale et al., (2001) who also reported that imidacloprid (Gaucho 70 WS @ 5g/kg) as seed treatment was found effective for the control of sucking pests of cotton. Present findings are in agreement with that of Afzal et al., (2002) who reported imidacloprid 25 WP @ 200g/acre as the most effective for the control of white fly. The present findings are in contradiction with Mandal (2015) who reported that the treatments comprising seed treatment with thiamethoxam 70 WS @ $3 \mathrm{~g} / \mathrm{kg}$ seed followed by two foliar applications of imidacloprid 17.8 SL @ 0.4 $\mathrm{ml} / \mathrm{L}$ at 15 days interval was found superior over all other treatment and Dar et al., (2002b) who reported that endosulfan @ $0.07 \%$, monocrotophos@0.04\%, dimethoate @ $0.03 \%$, NSKE @ 5\% alone and in combination were effective and economic control of insect pest complex of green gram. They also reported that Dimethoate in combination with NSKE (5\%) was found to be the most effective against whiteflies and jassids, while endosulfan proved to be the best against pod borers. NSKE $(5 \%)$ in combination with all the insecticides included in the study showed increase in yield compared to when used alone.

\section{Acknowledgments}

The authors are thankful to the Head, Division 
of Entomology, SKUAST-Jammu for providing all necessary facilities for experiments and nonteaching staff, Division of Entomology, SKUAST-Jammu for providing all necessary support.

\section{References}

Afzal, M., Ahmad, T. and Bashir, M.H. 2002. Relative toxicity of different insecticides against whitefly, Bemisia tabaci (Genn.) and black thrips, Caliothrips indicus on NM-92 mungbean, vigna radiate (L.). Pakistan J. Agr. Sci., 39(3): 224-225.

Dandale, H.G., Thakare, A.Y., Tikar, S.N., Rao, N.G.V. and Nimbalkar, S.A. 2001. Effect of seed treatment on sucking pests of cotton and yield of seed cotton. Pestol, 25(3): 20-23.

Dar, M.H., Rizvi, P.Q. and Naqvi, N.A. 2002b. Efficacy of neem and synthetic insecticides for the management of insect pests of green gram [Vigna radiata $(\mathrm{L}$. Wilczek]. Pest Manag. Econ. Zool., 10(1): 57-60.

Denholm, I., Cahill, M., Dennehy, T.J. and Horowitz, A.R. 1998. Challenges with managing insecticide resistance in agricultural pests exemplified by the whitefly Bemisia tabaci. Philosophy of Transaction of Royal Society (London B), 353(1376): 1757-1767.

Denholm, I., Devine, G.J., Gorman, K. and Horowitz, A.R. 2003. Insecticide resistance in Bemisia: a global perspective. Annals of the $3^{\text {rd }}$ International Bemisia Workshop, pp. 113.

Lal, S.S., Dias, C.A.R., Yadava, C.P. and Singh, D.N. 1980. Effect of sowing dates on the infestation of Heliothis armigera (Hub.) and yield. Int. Chick. News, 3: 14-15.
Mandal, S.K. 2015. Bio-efficacy of novel insecticides against whitefly, Bemesia tabaci in Mung bean. Ann. Plant Prot. Sci., 23(1): 16-18.

Marimuthu, T., Subramanian, C.L. and Mohan, R. 1981. Assessment of yield losses due to yellow mosaic infection in mungbean. Pulse Crops News, 1: 104.

Michaud, J.P. and McKenzie, C.L. 2004. Safety of a novel insecticide sucrose octanoate to beneficial insects in Florida citrus. Fla. Entomol, 87: 6-9.

Mohan, M. and Katiyar, K.N. 2000. Impact of different insecticides used for bollworm control on the population of jassid and whitefly on cotton. J. Pest. Res., 12(1): 99-102.

Oliveira, M.R.V., Henneberry, T.J. and Anderson, P. 2001. History, current status, and collaborative research projects for Bemisia tabaci. Crop Prot., 20: 709723.

Osorio-Diaz, P., Bello-Perez, L.A., SayagoAyerdi, S.G., Del Pilar Benitez-Reyes, M., Tovar, J. and Paredes-Lopez, O. 2003. Effect of processing and storage time on in vitro digestibility and resistant starch content of two bean (Phaseolus vulgaris) varieties. J. Sci. Food Agr, 83: 1283-88.

Singh, D.P. and Ahlawat, I.P.S. 2005. Greengram and blackgram improvement in India: past, present and future prospects. Indian J. Agr. Sci., 75: 243250.

Tikko, R.K. 1996. Distribution and management of insect pests of greengram and blackgram with special reference to life table studies of Spilosoma oblique Walker. M. Sc. Thesis, SKUAST, pp. 4759.

\section{How to cite this article:}

Nadeya Khaliq, Virender Koul, Uma Shankar, Suheel Ahmad Ganai and Thanlass Norboo. 2017. Bio-efficacy of Certain Selective Insecticides against Whitefly, (Bemisia tabaci) on Mungbean, (Vigna radiata L., Wilczek). Int.J.Curr.Microbiol.App.Sci. 6(7): 2344-2351. doi: https://doi.org/10.20546/ijcmas.2017.607.335 